\title{
Usage of Drones in Sports Communication - New Aesthetics and Enlargement of Space
}

\author{
By Andreas Hebbel-Seeger ${ }^{*}$ \\ Thomas Horky \\ Cora Theobalt
}

\begin{abstract}
Starting with a brief introduction to technology of video drones, the potentials of the use of this technology are discussed in the context of sports communication. Apart from many advantages like new perspectives and spectacular pictures by the view from above there are some other interesting aspects concerning the usage of drones like reciprocal effects or security and legal aspects which get more and more relevant. The contribution discussed these controversial aspects by balancing chances and risks of usage of drones in sports. In the sense of "Design Based Research" (Reinmann, 2005) the discussion is based on selected results of an empirical accompanying research on a cooperative project with the Sports Department of the City of Hamburg related to Hamburg Year of Aquatic Sports 2014 as well as on a visitor survey during the final of the German national sailing league 2014. In conclusion the paper suggests high acceptance and appreciation regarding the application of video drones within a sports and event context on the user side.
\end{abstract}

Keywords: Drones, journalism, communication, sports, fpv, immersion.

\section{Introduction}

In our digitized society the use of drones in sports communication becomes increasingly relevant. Goldberg et al. (2013,3) even speak of a "generation drone". The majority of literature on drones, however, focuses on ethical and legal aspects regarding their use in public spaces (Boucher, 2014; Gynnild, 2014). Moreover, drones are often associated with the military (Roush, 2014), journalistic purposes (Captain, 2012) or privacy and data protection issues (Boucher, 2014).

Aspects for the usage of drones or possibilities for enlargement of space and new aesthetics in sports are very rare to find. Gynnild $(2014,341)$ points out: "The innovation of drones for journalistic purposes will most likely replace or, more precisely, supplement visual news coverage on the ground with new kinds of aerial views as well as options for aerial close-ups that were formerly unseen." Goldberg et al. $(2013,24)$ emphasize possible reciprocal effects: "Since the application of drones in journalism is only just emerging, it is unknown how the public will react to their usage."

\footnotetext{
* Professor, Macromedia University of Applied Sciences, Germany.

${ }^{\dagger}$ Professor, Macromedia University of Applied Sciences, Germany.

* Macromedia University of Applied Sciences, Germany.
} 
In order to address these shortcomings in research identified by Goldberg at al. (2013), this article starts with a brief introduction of the technology used within video drones, as well as the contexts of usage for drones in sports. Its focus then resides on the results of two target group surveys on expectations and experiences with drones that were conducted in Germany (cf. HebbelSeeger, i.p.).

\section{Technology}

Photo and video drones are unmanned flight systems that carry a camera according to purpose and that are able to record and transmit footage. They are operated from the ground by means of a remote control. In addition, their steering is supported through electronic auxiliary systems such as flight stability systems. Concerning their general construction (especially with regards to the number of rotors) one can differentiate between different systems. Corresponding to the English term, the German Air Traffic Order (LuftVO) as well as the German Air Traffic Act (LuftVG) treat drones as "unmanned aerial vehicles". Due to the fact that the term "drone" has a negative connotation, resulting from its military purposes, both in the German and the English language it is also referred to as "multicopter". Multicopter depicts the umbrella term for all flight systems that use more than two rotors on one level for uplift and propulsion (Rattat, 2015).

The technology that is used in multicopters is the same as in modern smart phones. Integrated sensors such as an altimeter, motion sensors, gyroscopes and a GPS receiver enable an automatically stabilized and, if applicable, autonomous flight. The gyroscope determines the multicopter's position in a room by means of a 3-axes metering. By combining the data captured by the sensors, a 6-axes metering is created. Subsequently, a processor within the drone uses the aggregated data to calculate the drone's tilting, motion speed and rotation. On this basis, the drone is able to calculate its position and location in a room.

The technology relieves the pilot with regards to device control (Altmann, 2013, p. 28): Without interference the devices are able to react to varying environmental impacts, such as wind gusts, and to retain their position concerning height, width and length. Furthermore, complementary sensors, for instance ultrasonic transmitters and receivers, enhance GPS localization and allow for automatic orientation/distance measurement, e.g. to the ground. Thus, the sensors can be used to identify obstacles and to avoid collisions, for example with trees, bridges, etc.

Multicopters are usually equipped with a digital on-board control system (Naza), that serves as a flight-stabilizing support for the (remote) control. Moreover, it functions as a basis for the fail-save-system that is supposed to initiate an autonomous landing of the drone in case, for instance, the control signal is interrupted. Usually, the drone's control system is operated via a 2.4 $\mathrm{GHz}$ frequency. Since this frequency is commonly used by other systems such 
as mobile phones and wireless computer networks, there is the potential danger that the remote control fails or interferes with other signals. To guarantee secure operating, drones are thus equipped with a so-called "frequency hopping module" that switches among frequencies with minimum latency among a maximum range of channels.

The installation of photo and video cameras initially depends on the potential maximum load of the employed multicopter. A permanent installation at the aerial system is not recommended as it would lead to a severe restriction in pictorial design possibilities while the system's vibrations would furthermore be directly carried over to the camera. Due to its delay a cardan suspension does not present a satisfactory solution either. As a result, the usage of motor-controlled camera mountings (gimbals), which compensate the movements of the multicopter by means of a Naza interface, is inevitable. Moreover, according to device, they enable a remote-controlled tilting and turning of the camera on various levels (Tremayne \& Clark, 2014).

In addition to external control within sight, the digital image data of the installed camera can be broadcasted to a monitor or video glasses on the ground. On one hand, this is essential for controlled recording supervision, for which one constantly needs to review the size and arrangement of picture elements. On the other hand, especially the usage of video glasses enables highly immersive flight experiences. By giving the user the possibility to observe the drone's surroundings through the lens of the flying camera, he/she is provided with a first person view (FPV) (Altmann, 2013, p. 26-35; Bristeau et al., 2011).

\section{Usage in Sports}

Video drones are considered to be innovative alternatives to "real" helicopters, crane systems, spider cams and camera dollies. They are more flexible in usage and comparably inexpensive. Other than "real" helicopters, video drones create less downwash, which is usually caused by movements of the rotors. Moreover, they are considerably less noisy and, not least due to their smaller size, are also more maneuverable so that they can be operated closer to the point of action, amongst other things. On grounds of these circumstances, "large" helicopters need to keep a relatively long distance to the filmed object which calls for large focal lengths or zoom factors that result in narrow angles of view. Video drones, in contrast, are able to picture a room in a different manner by closely approaching objects and thus they mostly forgo zoom (Landsiedel, 2015). In doing so, video drones can "interact" with stationary or flying constructions with regards to pictorial presentation, for example by flying underneath bridges, through buildings or by "dipping" into urban canyons. However, higher flight levels and, most of all, longer flight durations as well as longer distances so far remain reserved to "large" helicopters (ibid.).

As part of rapid technological developments, the performance of unmanned aerial vehicles improves within shorter and shorter cycles. 
Subsequently, acquisition costs are decreasing - especially for beginner systems - which helps to extend the market, accelerates innovation in this area and lets product costs become more consumption-friendly. While it was specialized production companies at first that (also) used video drones for sports communications, by now not only the number of service providers in this area has greatly increased but also the usage of drones in a semiprofessional sports environment. By using their own flying devices, soccer coaches, for instance, can monitor their team's practice, athletes are enabled to improve their own motion perception through the visualizations of their movements (Higuchi et al., 2011), rowing coaches are able to create studies on technique from the air or event organizers themselves can provide their audience with aerial footage. The reasons for this behavior are obvious: video drones open up a new perspective for documentation as well as practice purposes.

Organized sports were caught off guard by this development. Guidelines regarding the usage of video drones in practice situations and competitive environments, or provisions for unmanned aerial vehicles are mostly missing within sports rulebooks. In Germany, this is where the aviation law sets in: It sets the general framework which particularly forbids the flying over crowds. Nevertheless, there is an urgent need for action to not only address security related aspects but particularly ensure the non-impairment of equal opportunity in sports. What has to be ruled out is that single teams make use of (video) drones as tactical whistleblowers or pace makers. Because in situational sports, outside of standardized sports facilities, an elevated position makes it possible to identify not only advantageous water areas or terrain but also competitors in larger starting areas. Through the drone's position and/or movement in the air a forwarding of external information becomes possible, which could be relevant for decision-making in the respective sport.

\section{View from Above}

In sports communication, a view from above generally provides overview. By observing the athletic happenings from distance from above, the spatial, tactical and situational dimensions of events on the grass, water or mountain disclose themselves to the spectators. The spatial dimension is dominated by aesthetic aspects that communicate the athletic happenings in relation to their environment: type of sport, athletic happenings and (e.g. urban) space blend into one communicative entity (cf. Figure 1). A view from above illustrates the decision to pass into "open space" within a soccer game as well as a sailing maneuver in a competition with other boats, whose relative position becomes as identifiable to the consumer on screen as the situational conditions of a decision (e.g. distance to shore, wind fields, etc.) (cf. Figure 2). Along these lines, a "view from the ground ... is characterized by a shortage, namely a shortage in knowledge and the associated power" (Reiffers, 2013, pp. 12, own translation). Through a view from the ground the audience is involved in the 
happenings, yet without being able to extrapolate what is seen in all dimensions. Thus, the view from above is more than "simply" a new perspective. "Through a view from above the subject buys the impression of comprehensible order and own power through distance (Reiffers, 2013, p. 11, own translation). The outlined presentation possibilities of sports in space thus go beyond the actual subject area and are broadened by a psychological component. The view from above does not only open up an unfamiliar perspective with own aesthetics that simply serve the intermediation of a complete situational event. But even the perspective itself implicates to the audience the feeling of being better informed and having more information: "The metaphor of overview combines the structured concepts of knowledge and of power with the view from above and puts it in contrast to the view from the ground" (Reiffers, 2013, p. 17, own translation).

Figure 1. Swimming Start of the Triathlon World Cup 2014 in Hamburg from the Perspective of a Video Drone

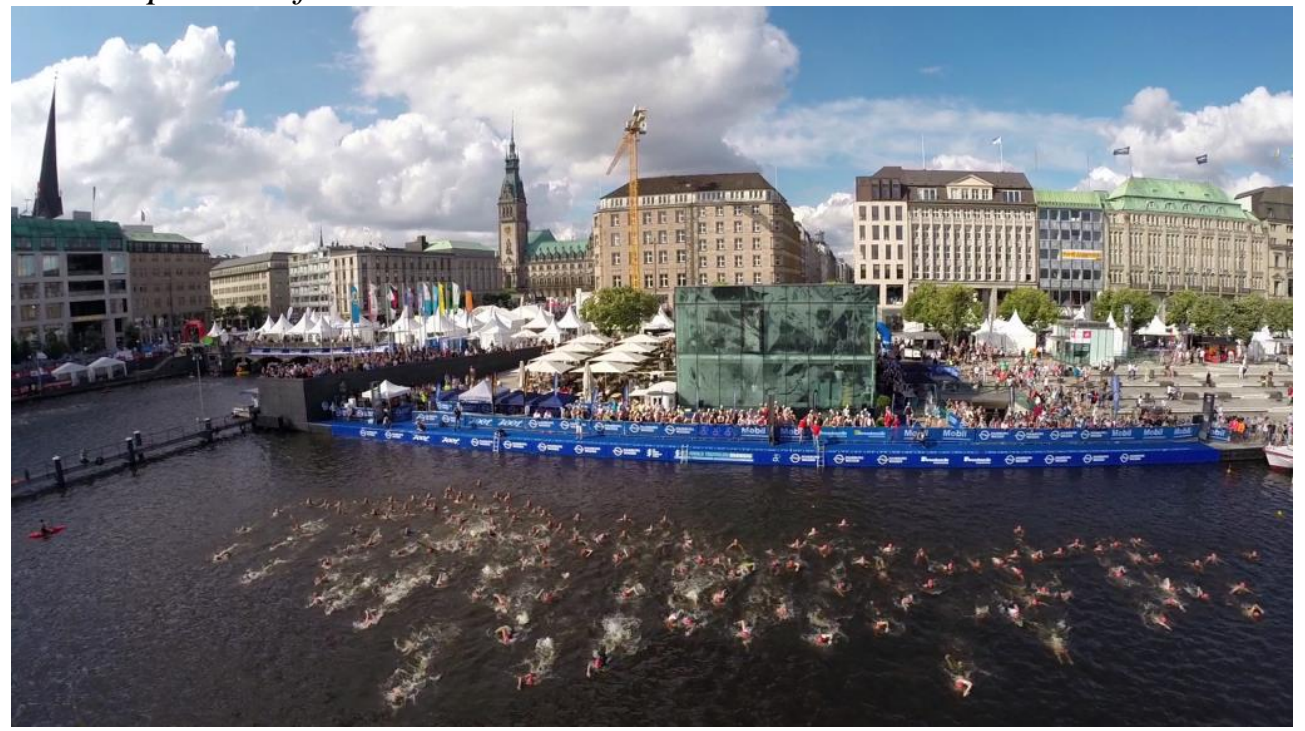

Photo: Hebbel-Seeger

Especially outside of normalized sports facilities, this view from above used to be accompanied by considerable effort: with the aid of helicopters and cranes on one hand and by longer distances to the happenings (filmed from a helicopter) as well as a more rigid fixation of the filming location (from a crane) on the other hand. Due to their notably smaller dimensions, high agility and an uncritical downwash of the rotors (Reiffers, 2013, p. 17, own translation), drones allow for considerably lower flight levels in close proximity to the (athletic) happenings. For one, the possibility to witness dynamic happenings almost from the perspective of an opponent or teammate leads to an immersive experience for the consumer ("Immersion means becoming physically or virtually a part of the experience itself" (Ermy \& Mäyrä, 2007, p. 40). For the other, even a visualization in difficult environments becomes possible, when video drones follow bicycle racers 
through urban canyons or accompany snowboarders over hazards and through obstacles (Tremayne \& Clark, 2014).

Figure 2. Regatta Communication by Means of a video Drone at the Final of the German National Sailing League in Hamburg 2014

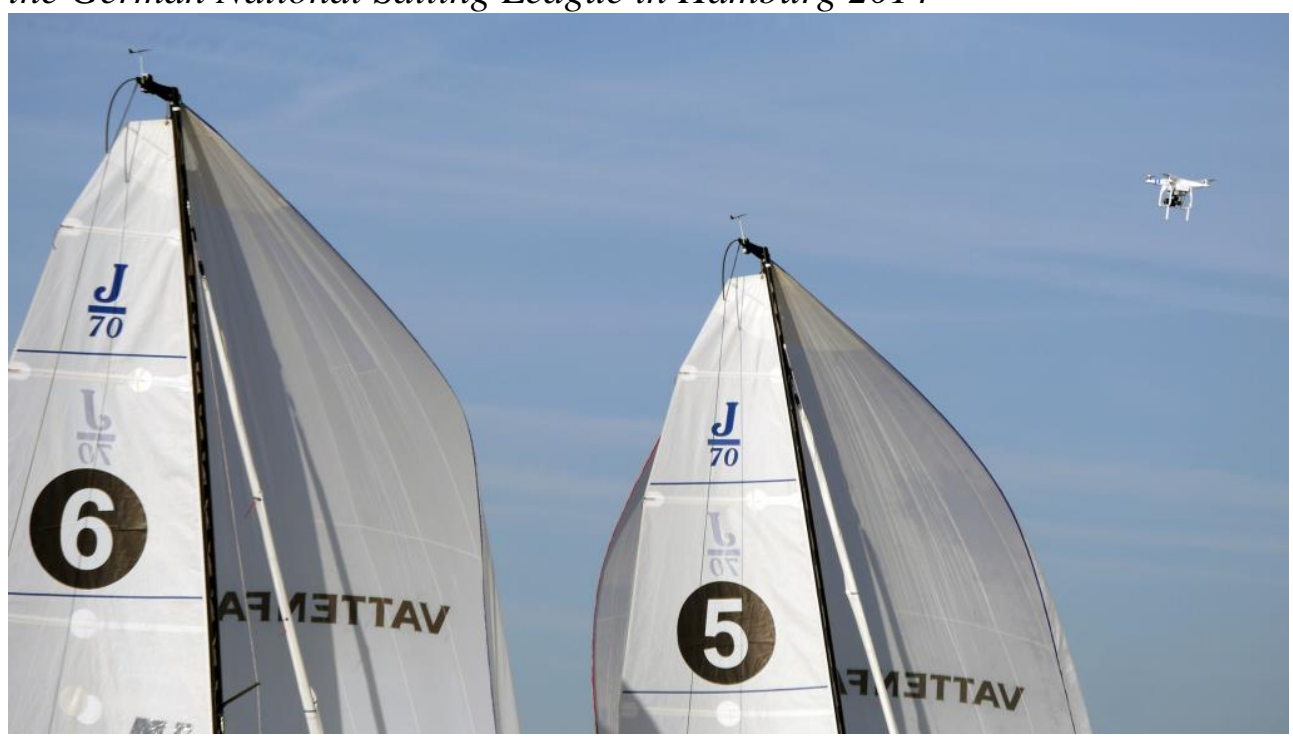

Photo: Hebbel-Seeger

\section{Online Survey}

Expectations towards and experiences with the reception and communication of (moving) image content, produced by video drones, was tested by means of a target group online survey in November 2014. The survey fell in line with the last "major" sporting event in Hamburg's "Jahr des Wassersports" ("Year of Aquatic Sports"), the final of the German national sailing league.

The hyperlink to the survey was communicated via the Facebook page of Hamburg's Year of Aquatic Sports. Simultaneously, the Facebook page of the "Deutsche Segel Bundesliga (DSBL)" (German national sailing league) was used to invoke a participation in the survey. During the evaluation period between November $10^{\text {th }}, 2014$, and November $25^{\text {th }}$, 2014, out of 440 participants 347 subjects answered the survey, which equates a net participation of 78.8 percent.

The participants' gender ratio of about one third women (36.34 percent) to two thirds men (63.66 percent) reflects the respective distribution among the Facebook fans of Hamburg's Year of Aquatic Sports with about 35 percent to 64 percent. What is more, it also corresponds to the gender ratio within organized sailing in Germany (34 percent female versus 66 percent male members of the German sailor's association (Deutscher Segler-Verband), as of 2008) (Seglerrat im DSV, 2009). Regarding gender distribution on the Facebook page of the national sailing league, there is no data available. 
The referenced data does not provide any indication that the technology focus of the topic (video drones) led to a gender-dependent shift of the participation of the online survey. Similarly, the survey data does show any signs of gender-specific discrepancies within the answers. Thus, it was abstained from applying a corresponding differentiation within the display of the results hereafter.

The same applies to differentiation according to age groups. The distribution with respect to the age of the participants ranges throughout all appointed clusters. The distribution to different age groups does not allow for a differentiated observation.

The distinct majority of subjects (79.6 percent) had already seen content produced by video drones at the time of the survey. Only 12.7 percent negated the question while 7.7 percent of the participants were not able to determine whether they had seen respective content or not. The inquired differentiation among the communication channels TV and online, however, is not very distinct as the majority of public and private TV channels in Germany uses the Internet as a distribution channel in parallel with cable, satellite and terrestrial TV. Besides current TV hybrid devices, peripheral devices such as "Apple TV" or Google's "Nexus Player" lead to a consumption of web TV offerings via the usual television set. Accordingly, the survey results on the distribution channel or channels (multiple answers possible), via which the subjects encountered drone content, only provide indications on one unsurprising insight, at best: innovative media formats, such as the production of audio visual content by means of video drones, enter online communication much quicker than a traditional medium such as television (cf. e.g. Brem et al., 2011).

The importance of live communication as field of application and touch point for video footage from the air seems to be comparatively high, as it was mentioned 90 times and thus by about 34.5 percent of those participants, who previously stated that they had already seen respective content. Nevertheless, one needs to take into consideration that video live streaming, amongst others with footage of video drones, constitutes an essential element of the DSBL not only in online but also in on-site communication. Since the placement of the hyperlink to the online survey on the Facebook pages of Hamburg's Year of Aquatic Sports as well as the DSBL - not at least due to the difference in basic population - predominantly addressed those interested in sailing and the DSBL as before mentioned, it can be assumed that only the possibility to experience an according live event in one's "own" sport lead to the respective demand.

Following the distribution logic of the online questionnaire via the Facebook pages of Hamburg's Year of Aquatic Sports and the DSBL, it does not surprise that sailing in particular as well as aquatic sports in general dominate the list of sports (multiple answers possible), for which the subjects believe they have seen content produced by video drones. Noteworthy, with regards to the question about sports that are being visualized with the aid of drones, were the topics that were not mentioned in the responses: On occasion of the Olympic winter games in Sochi 2014, which only dated back about half a year at the time of the survey at hand, video drones were systematically 
employed during the coverage of the skiing freestyle competitions in the "Extreme Park". Images that were distributed globally and in Germany even via public television channels. Yet, no survey participant mentioned winter sports as a sport that already draws on video drones for content production. Potentially, this content was indeed not inquired by the subjects. It is also conceivable, however, that this particular form of image creation via video drones was not recognized among the variety of alternating recording devices (spider cams, crane cameras, etc.) and/or that the different perspectives were combined into a harmonized composition during production, so that existing differences were levelled in the overall picture.

Figure 3. "If you have Already Experienced the Usage of Video Drones at a (sporting) Event: Do Unmanned Aerial Vehicles in Action cause you to be Frightened?" (data in absolute numbers, $n=326$ )
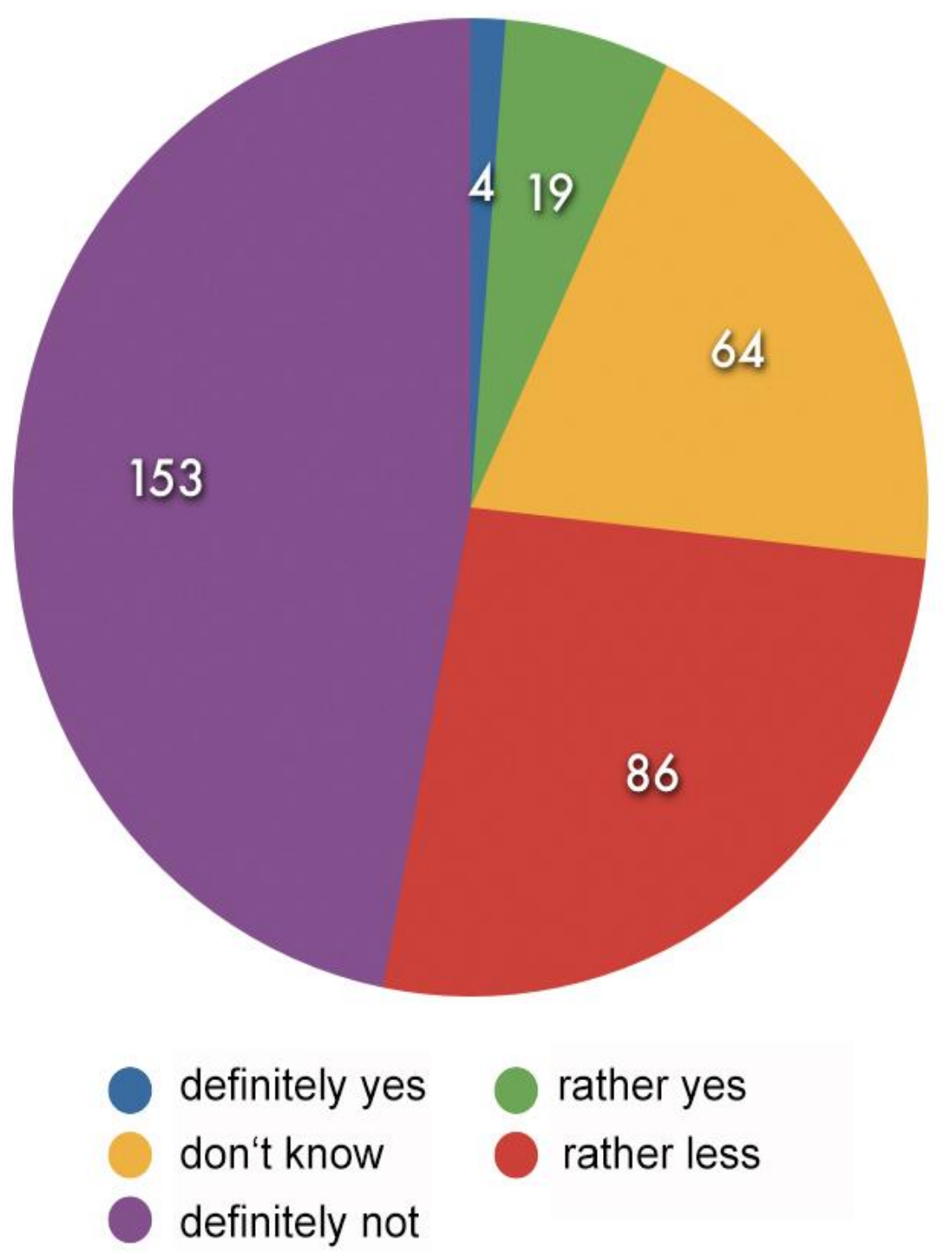
Provided that the recordings of video drones in sports communication were indefinable as such for the subjects, the majority of participants reviewed the appeal of the footage as positive: Over 90 percent of the participants stated that they watched respective content "very gladly" (51.7 percent) or "rather gladly" (39.7 percent). An assessment of the extend to which aspects of novelty and curiosity played a role alongside the specifics of pictorial design (Berlyne, 1974; Edelmann, 1996, p. 364) is not possible.

Figure 4. "If you have not Experienced the Usage of Video Drones at a (sporting) Event Yet: Do you think that Unmanned Aerial Vehicles in Action would Cause you to be Frightened?" (data in absolute numbers, $n=64$ )
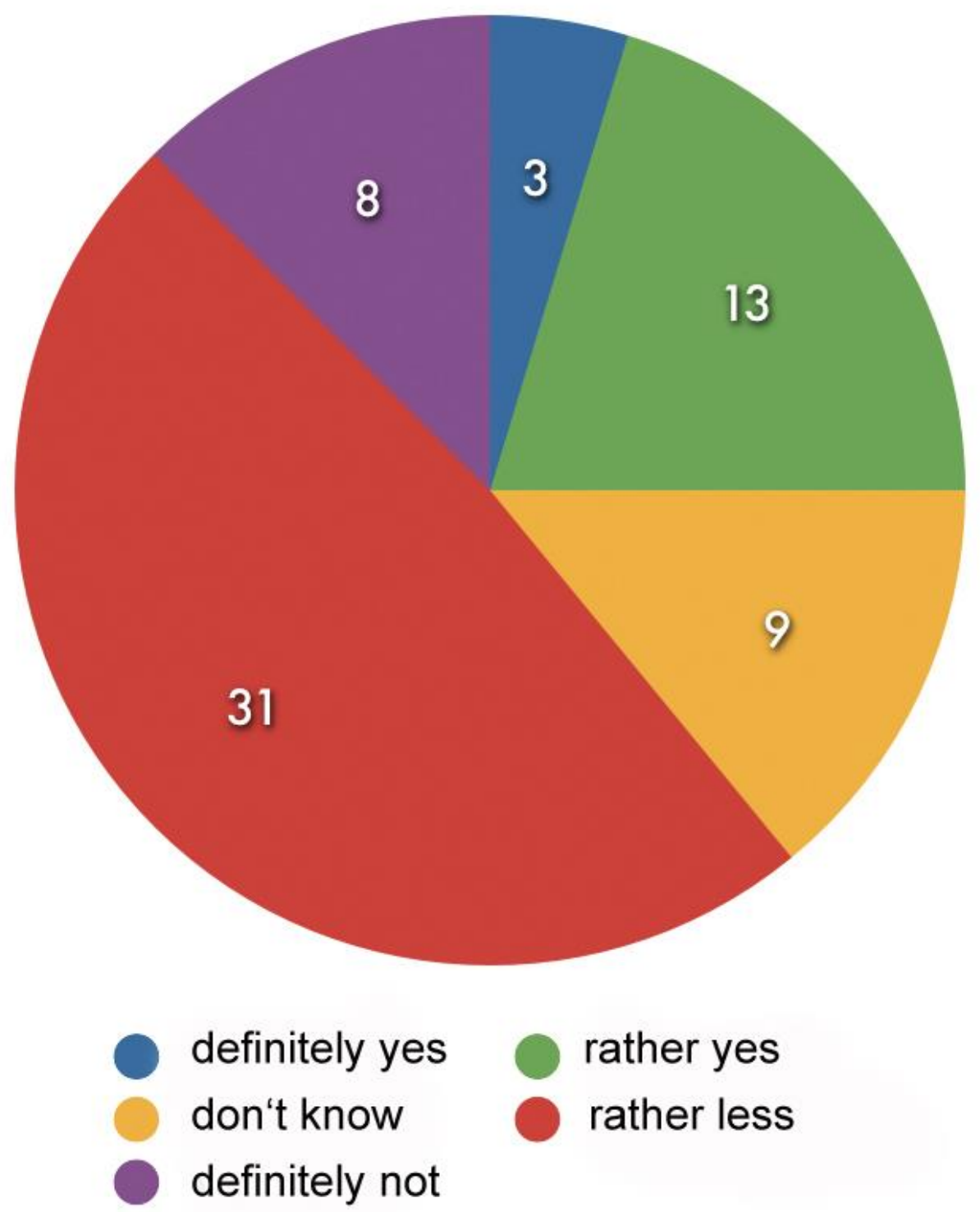
The positive assessment of the production output on part of the respondents is opposed with little awareness for the potential dangers of the application of video drones (cf. Figures 3 and 4). Differences in response behavior with regards to feelings of anxiety among participants, which had already experienced the usage of a video drone live, and those that had not, were below the threshold of materiality of a one-way analysis of variance $(t$ test).

Figure 5. "Which Associations/impressions/expectations do you Generally have Regarding Video Drones in Sports?" (multiple answers possible, data in absolute numbers, $n=325$ )

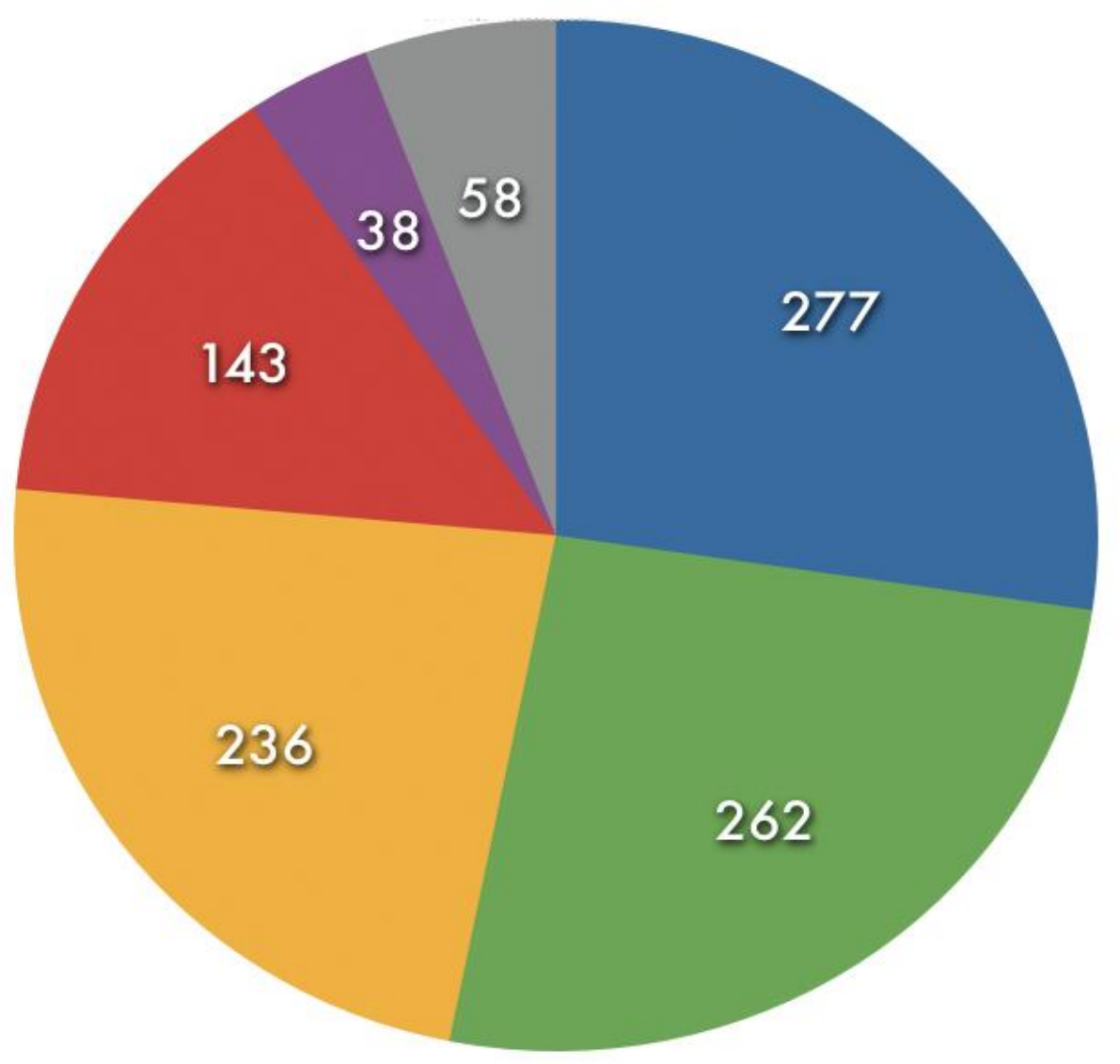

spectacular images

new perspectives

overview of the (sports) events

innovation

crash danger

surveillance 
Moreover, the respective numbers of responses reveal an overlap in responses. Despite the fact that the question explicitly distinguished between "already experienced" and "not yet experienced", numerous respondents answered both questions. That the risks of application of video drones play a minor role in the perception of the participants overall becomes evident from the associations, impressions and expectations connected to video drones by the respondents. Despite the possibility to choose multiple responses, the negative aspects of "risk of failing" and "surveillance" was mentioned by less than a third of the respondents (cf. Figure 5). At that, the participants seem to doubt the technical reliability of unmanned aerial vehicles less than potential misuse. The respondents did not exert the possibility to contribute additional aspects to the question.

Figure 6. "To what Extend do you Think that the Application of Video Drones has a Positive Effect on the Overall Quality of Sports Coverage?" (data in absolute numbers, $n=323$ )
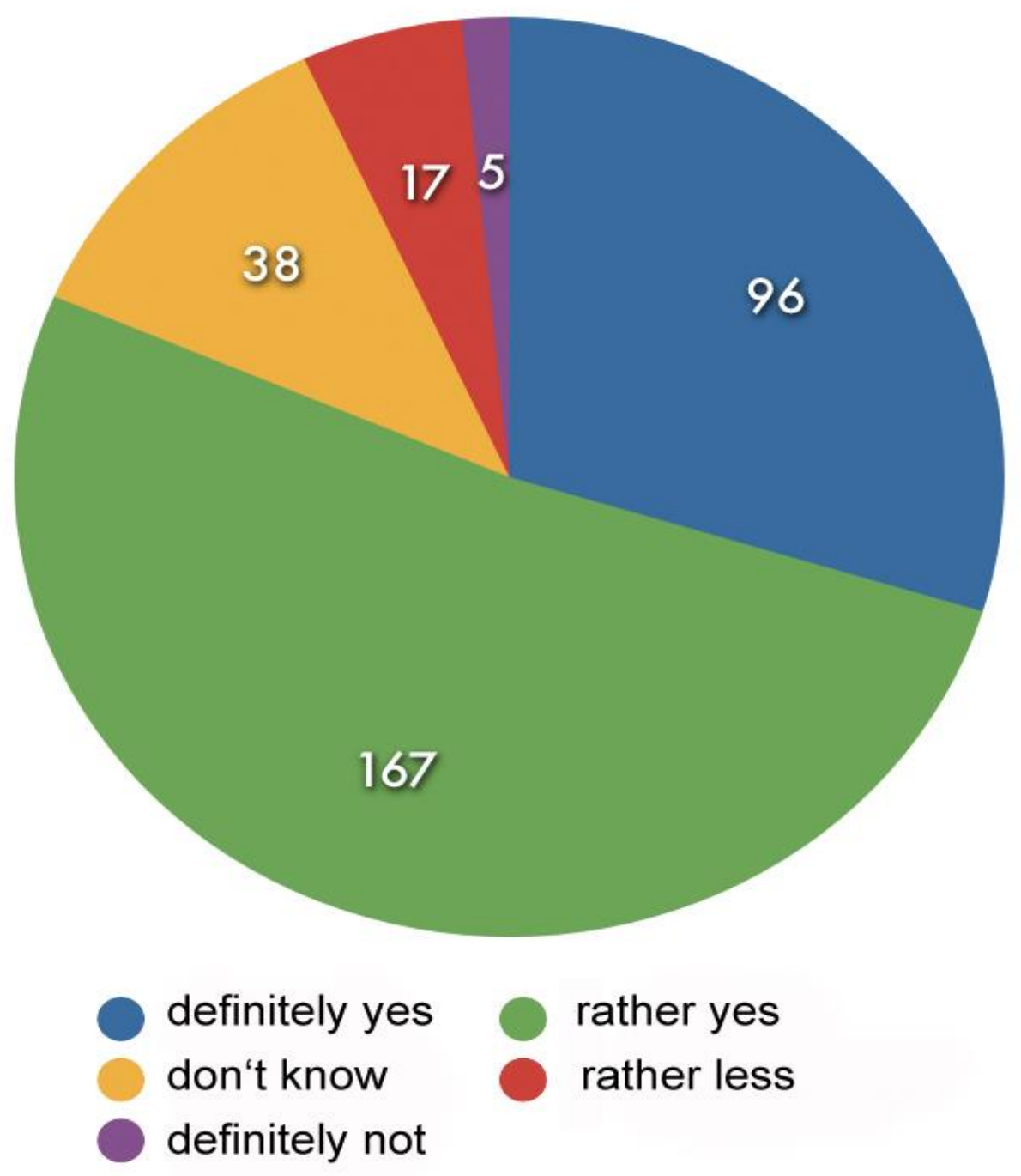
Thus, it seems logical to assess that the respondents expect positive effects of the application of video drones on the overall quality of sports coverage (cf. Figure 6).

Due to the peculiarity of comparatively long distances and free movement in sailing competitions - compared to competitions in the "classical" disciplines of rowing or canoeing where opponents move within clearly defined, parallel tracks - the anticipated consequences of video drone usage on the quality of sailing coverage in particular was of interest to this survey (Figure 7).

Figure 7. "To what Extend do you Think that the Application of Video Drones has a Positive Effect on the Quality of Sports Coverage with Regards to Sailing?" (data in absolute numbers, $n=323$ )
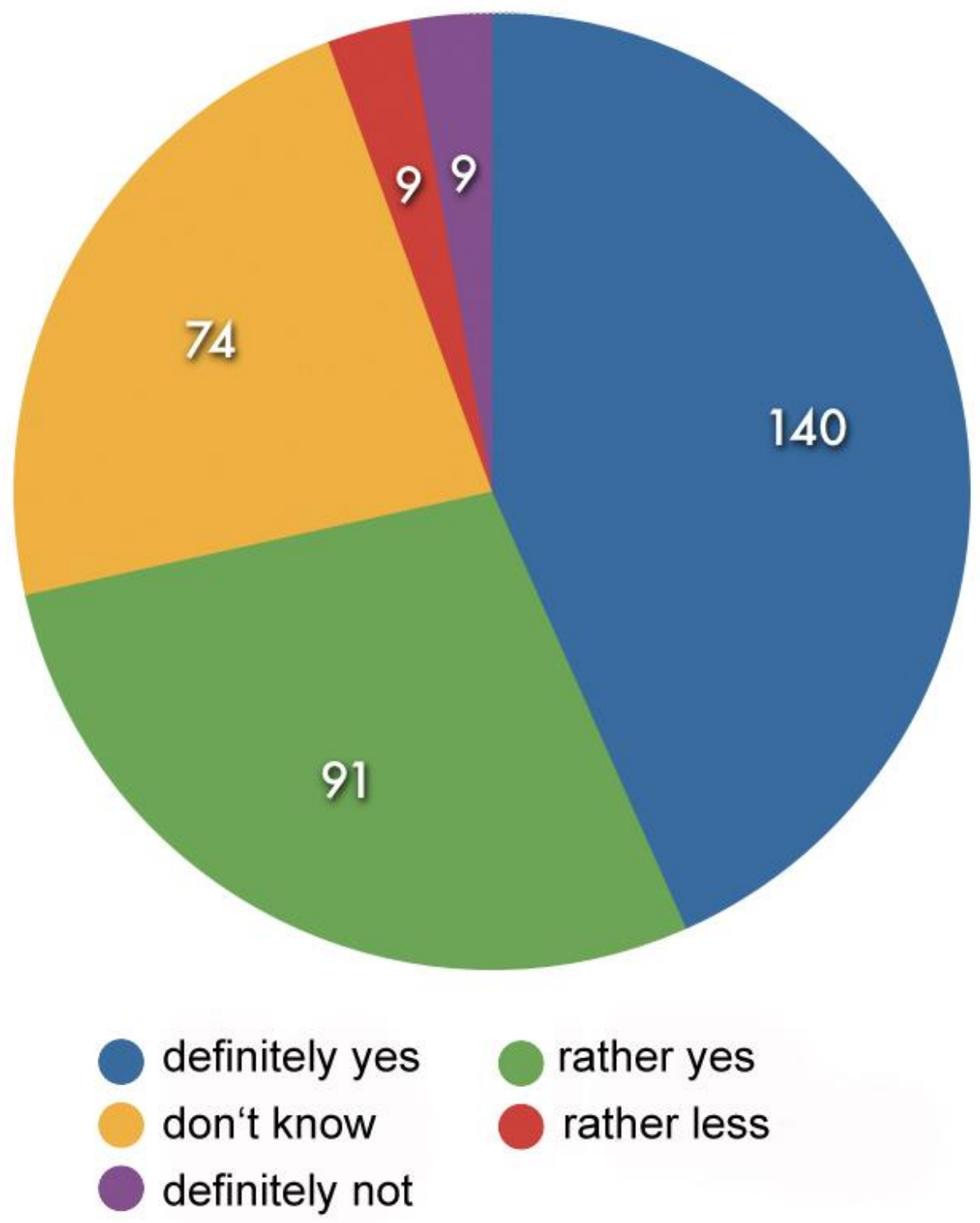

In fact, noticeably more respondents expect "very strong" effects of the 
application of video drones on the quality of sports coverage with regards to sailing in particular than with regards to aquatic sports in general. Simultaneously, the number of participants who did not want to commit to an opinion ("I don't know") nearly doubled.

This result can be interpreted as an evidence for the hypothesis that sailing can benefit more from a visualization of the sporting events from above than other (aquatic) sports: The respondents are persons with an interest in aquatic sports, which is yet based on experiences and affinities depending on the sport. Accordingly, a cross comparison was applied to relate the participants' statements on personal affinities in aquatic sports to the expressed expectations regarding the quality change of coverage through the application of video drones. This lead to the interpretation that respondents with sailing relations assess the role of video drones for communication of central aspects of sailing competitions (order of the boats, tactical decisions, realization of practical sports techniques/maneuvers, etc.) as "very important" since there is almost no alternative in order to disclose these happenings from the outside. Unaware of the situation of communication within other sports, the same respondents answer respectively more careful and believe "rather yes" in positive effects.

In reverse, the potential value added of video drones does not seem to be as remarkable in other sports as sailing, insofar that they don't carry over to people without pertinent expertise. As a result, indecision ("I don't know") with regards to a generalized reflection of application scenarios substitutes guarded optimism, which would give reason to "rather yes" expect quality improvements.

\section{Visitor Survey}

Concerning the application of video drones for event communication by means of online media, there is a spatial and, where applicable, temporal distance between the usage of a device at an event on one hand and consumption by the consumer on the other hand. Only the media products in the form of photos and/or videos are communicated, while the drone as production device stays behind the content and is only implicitly identifiable via the type of content or the drone-specific perspective.

A live audience, however, can experience a video drone beyond the produced and displayed (e.g. via video walls) content: visually as a flying device in the sky as well as aurally through the production of noise of the device. The footage created by video drones yet serves further purposes than distance-overcoming (online) communication within the context of events: Within an event context, the employed media are supposed to contribute to the overall experience. Whereas in distance-overcoming communication, the media themselves become the focal point of the experience.

If and, if applicable, to what extend the expectations, acceptance and assessments regarding the usage of video drones differ among visitors and persons, which encounter the same content as part of mediated communication 
(e.g. via TV or online), was investigated by means of an audience poll at the final of the German national sailing league on October $31^{\text {st }}$ and November $1^{\text {st }}$, 2014.

An electronic questionnaire was created for the poll, which was then handed over to the visitors of the event via a tablet computer (iPad) and was completed by 58 subjects overall. The sample is composed of 26 women and 32 men aged 18 to 63 years. A little more than half of the respondents $(n=31)$ is interested in sailing. Out of those not interested in sailing, 14 respondents were at the venue due to a walk and thus by mere coincidence, eight respondents attended because of personal acquaintances with active athletes involved in the local event and another five persons accompanied fans of the event.

As part of the mentioned event, live footage of the races was displayed on small screens in addition to the happenings that could be observed from the landing stage and to live comment broadcasted via loudspeakers. Yet, the monitors were not viewable directly from the central spectators' area but were distributed at the venue itself. The full-video content displayed on-screen was an edited mix of live footage from three perspectives $\left(1^{\text {st }}\right.$ from an on-board perspective, $2^{\text {nd }}$ from the perspective of an auxiliary vessel and $3^{\text {rd }}$ from the air) and computer animations (SAP's "Sailing Analytics"). The content thus visualized the position of the boats on the water to one another and was completed by central movement data (speed, angle to the wind, etc.).

In accordance with the research interests of this paper regarding the expectations and experiences with "aerial content" and video drones, the first topic of interest during the questioning was the visitors' prior experience with the consumption of moving images and photos from the air within the context of sports and events. 36 subjects stated that they had already seen content produced by drones while 21 persons responded that they had not actively consumed such content before. One respondent was not able to answer this question with certainty. An inquiry about the subjective evaluation of the recordings of video drones, be it based on own experience or naïve expectations, lead one half to be undecided $(n=27)$ while 20 respondents rated the recordings as "good" and nine of the participants as "very good". Only 1 person judged the value of the content as "bad".

The title "drone" often implicates associations with a military or intelligence usage (Biermann \& Wiegold, 2015, pp. 7). What is more, flying devices generally constitute a physical danger in case of a crash or an uncontrolled movement: may it be due to technical mistakes, incorrect operation or a deliberate, felonious manipulation. While this aspect is only indirectly relevant for consumers of mediated content, visitors of a live event are directly affected. Nevertheless, a distinct majority of the polled visitors $(n=46)$ classified video drones as safe and stated that they do not have any fears concerning their usage. Only 4 of the participants were undecided whilst 8 respondents considered video drones in general to cause fear.

Other than with fixed camera systems, especially the recording activities and picture details of video drones are not always obvious to the visitor. Correlatively, it was of interest whether the respondents felt discomfort due to 
the application of flying cameras as one might, for example, feel observed. Admittedly, the majority of respondents does not have these doubts $(n=38)$. Still, 15 respondents do express these fears while 5 do not have an opinion on this topic yet.

The benefit of content produced by video drones for the presentation of sports in general and sailing in particular (since the poll took place in a sailing context), is regarded as "large" by a majority of the participants $(n=51)$. Merely 2 participants do not see a future perspective for video drone content and 5 respondents did not have a fixed opinion on the matter.

\section{Summary and Outlook}

Sport is produced as movement in space and, likewise but on a different level, communication is staged through movement in space. On that note, aerial shots produce unusual picture details and changes in perspectives which create a whole new effect for the observer. Yet, these aerial shots are not only characterized by single movements. Also the dialogue between movement and space constitute the essence of aerial footage, which is influenced significantly by camera angles and tracking shots.

With regards to the application of video drones for event communication by means of online media, there is a spatial and, where applicable, temporal distance between the usage of a device at an event and the consumption by the consumer. Only the media products in the form of photos and/or videos are communicated, while the drone as production device stays behind the content and is only implicitly identifiable via the type of content or the drone-specific perspective. For a live audience, however, a video drone can be experienced beyond the produced and displayed (e.g. via video walls) content: visually as a flying device in the sky as well as aurally through the noise production of the device. On top of that, the footage created by video drones serves further purposes than distance-overcoming (online) communication within the context of events: Within an event context, the employed media are supposed to contribute to the overall experience, whereas in distance-overcoming communication the media themselves become the focal point of the experience.

The results of the online survey that were presented within the scope of this paper suggest high acceptance and appreciation regarding the application of video drones within a sports and event context on the user side. At the same time, the survey results reflect the user behavior, which was also analyzed yet not explained in more detail within this paper: Independent from the respective temporal placement within an event context (pre, live and post coverage) and from the provenience (unedited sequences vs. edited videos), reports with integrated or linked aerial content were inquired more often and were shared more frequently with others.

One of the reasons for this behavior can be found in sport as a subject matter itself. Visualization from the air does not "only" provide a new perspective. Instead, only a view from above often makes relevant connections, 
for example regarding the interpretation of a match situation, accessible to the audience. Another motivation, at least thus far, probably is inquisitiveness since the well-known can now be experienced in a new way. Similar to the by now familiar - perspective at the "point of action", mediated through onboard and on-person cameras, it is to be expected that the last-mentioned aspect will soon become an end in itself and thus less important. At the same time, the systematic exploration of specific value added for event communication supported by video drones will gain more importance.

The exploratory poll of a live audience confirmed the results of the online survey with regards to user expectations. Real physical dangers for a live audience evidently affected the general attitude of the respondents towards this new technology just as little as aspects regarding data protection law in live situations.

The exploration, localization and description of the application of video drones within the context of professional production and communication of sports events is not at least driven by the trivialization of drone technology and the associated mass distribution.

Without addressing the media-ethical and legal implications of video drone usage, that need to be discussed elsewhere, necessary differentiation of video content will soon only be accomplished through the quality of pictorial design and good coordination within the communication mix and no longer via the mere type of content (from the air or not).

\section{References}

Altmann, R. (2013). Mit der fliegenden Kamera fotografieren. C'T digitale Fotografie, 3, 20-41.

Berlyne, D. E. (1974). Konflikt, Erregung, Neugier. Zur Psychologie der kognitiven Motivation. Stuttgart: Klett.

Biermann, K. \& Wiegold, T. (2015). Drohnen. Chancen und Gefahren einer neuen Technik. Bonn: Bundeszentrale für politische Bildung.

Boucher, P. (2014). Civil drones in society. Societal and Ethics Aspects of Remotely Piloted Aircraft Systems. Luxembourg: European Union. http://publications.jrc. ec.europa.eu/repository/bitstream/111111111/33061/1/civil\%20drones\%20in\%20 society\%20-\%20online\%20copy.pdf [03.03.2016]

Bristeau, P.-J., Callou, F., Vissière, D. \& Petit, N. (2011). The Navigation and Control technology inside the AR.Drone mirco UAV. http://cas.ensmp.fr/ petit/papers/if ac11/PJB.pdf [14.05.2016]

Brem, A., Gerhard, D., Gutt, C. \& Lettl, C. (2011). Innovationskommunikation: Theorie und empirische Untersuchung der externen Kommunikation von Innovationen über das Internet und sozialen Medien. Munich: Grin.

Captain, S. (2012). Livestreaming Journalists Want to Occupy the Skies with Cheap Drones. Wired. http://www.wired.com/threatlevel/2012/01/occupy-drones/ [03.03.2016]

Edelmann, W. (1996). Lernpsychologie (5. Auflage). Weinheim: Beltz PVU.

Ermy, L. \& Mäyrä, F. (2007). Fundamental Components of the Gameplay Experience: Analysing Immersion. In S. de Castell \& J. Jenson (Eds.), worlds in play: International Perspectives on Digital Games Research (p. 37-54). New York: 
Peter Lang.

Goldberg, D., Corcoran, M., \& Picard, R.G. (2013). Remotely Piloted Aircraft Systems \& Journalism Opportunities and Challenges of Drones in News Gathering. Reuters Institute for the Study of Journalism, Oxford University: Oxford.

Gynnild, A. (2014). The Robot Eye Witness. Extending visual journalism through drone surveillance. Digital Journalism 2, (3), 334-344.

Hebbel-Seeger, A. (i.p.). Video-Drohnen in der Eventkommunikation. In A. HebbelSeeger, T. Horky \& H.-J. Schulke (Ed.), Sport als Bühne - Kommunikation und Mediatisierung von Sport und internationalen Sportgroßveranstaltungen. Aachen: Meyer\&Meyer.

Higuchi, K., Shimada, T. \& Rekimoto, J. (2011). Flying sports assistant: external visual imagery representations for sports training. Proceedings of the 2nd Augmented Human International Conference (AH '11). ACM, New York, NY, USA, (Article 7, 4 pages).

Landsiedel, T. (2015). Mit der Drohne in die Schlucht. http://filmeundmacher.de/ 2015/06/15/mit-der-drohne-in-die-schlucht/ [03.03.2016]

Rattat, C. (2015). Multicopter selber bauen: Grundlagen - Technik - eigene Modelle. Heidelberg: dpunkt.

Reiffers, M. (2013). Das Ganze im Blick. Eine Kulturgeschichte des Überblicks vom Mittelalter bis zur Moderne. Bielefeld: Transcript.

Reinmann, G. (2005). Innovation ohne Forschung? Ein Plädoyer für den DesignBased Research-Ansatz in der Lehr-Lernforschung. Unterrichtswissenschaft, 33, 1, 52-69.

Roush, A. (2014). Up in the Air. The drone revolution isn 't coming - it's already here. Can UT expertise help us navigate the future? http://alcalde.texasexes.org/2014/ 10/up-in-the-air/ [03.03.2016]

Seglerrat im DSV, Arbeitsgruppe "Gender Mainstreaming" (2009). GenderMainstream in Segel-Vereinen. http://alt.dsv.org/fileadmin/user_upload/media/_ news/PDF/Microsoft_PowerPoint_-_Gender-Mainstreaming_SR_01-09.pdf [08.04.2015]

Tremayne, M. \& Clark, A. (2014). New Perspectives from The Sky. Unmanned Aerial Vehicles and Journalism. Digital Journalism, 2, 2, 232-246. 
